\title{
Non-Contact Method for Surface Roughness Measurement After Machining
}

\author{
J. Valíček ${ }^{1}$, M. Držík ${ }^{2}$, T. Hryniewicz ${ }^{3}$, M. Harničárová ${ }^{4}$, K. Rokosz ${ }^{3}$, M. Kušnerová ${ }^{1}$, \\ K. Barčová ${ }^{1}$, D. Bražina ${ }^{5}$ \\ ${ }^{1}$ Institute of Physics, Faculty of Mining and Geology, VŠB - Technical University of Ostrava, 17. listopadu 15/2172, \\ 70833 Ostrava-Poruba, Czech Republic, corresponding author: jan.valicek@vsb.cz \\ ${ }^{2}$ Institute of Construction and Architecture, Slovak Academy of Sciences, Dúbravská cesta 9, 84503 Bratislava, Slovakia \\ ${ }^{3}$ Division of Surface Electrochemistry and Technology, Koszalin University of Technology, Racławicka 15-17, \\ PL 75-620 Koszalin, Poland \\ ${ }^{4}$ Nanotechnology Centre, VŠB - Technical University of Ostrava, 17. listopadu 15/2172, \\ 70833 Ostrava-Poruba, Czech Republic \\ ${ }^{5}$ Department of Informatics and Computers, Faculty of Science, University of Ostrava, 30. dubna 22, \\ 70103 Ostrava, Czech Republic
}

\begin{abstract}
The paper deals with the measurement and identification of surfaces after machining in a non-contact manner. It presents a new modified measurement method and its implementation, the results of intensity distribution in the defocusing plane, their analysis and interpretation. The scanned intensity distribution at the defocusing plane gives information necessary to assess the second derivatives, and thus, surface functions which can be used to determine groove curvatures of the real surface morphology. The proposed method of measurement has proved to be very sensitive in evaluating the differences between surface finishing methods by which the measured surface standards (etalons) were machined. Two methods of machining were chosen: face grinding and planning. By comparing the roughness standard values $R a$, there were obtained relationships between these values and the parameter of the characteristic frequency of vertical inequality being measured according to the presented method. A good correlation between the measured and surface standard values with the correlation coefficient taking a range of values from 0.8 to 1 was achieved.
\end{abstract}

Keywords: Surface roughness, non-contact measurement, surface finishing methods

\section{INTRODUCTION}

$\mathrm{R}$ EQUIREMENTS for improving quality of metrological methods are increasing all the time and there is a growing need to develop the most accurate control techniques. A development of the commonly used stylus instruments has achieved a high technical level. One of the main disadvantages of these mechanical profilometers is that a tip must be in mechanical contact with a measured surface but this requirement leads to potential risk of soft material damage. Their accuracy and sensitivity are limited by unwanted resonance frequencies of the measuring tip as it moves over the surface. These disadvantages can be basically eliminated by using non-contact measuring devices which can be developed on the basis of optical principles. In addition, optical measurement methods may also eliminate an excessive selectivity of results and provide more comprehensive information regarding a state of the surface profile. A number of non-contact optical and also nonoptical methods have been developed [1], [2], [3], [4], [5], [6], [7]. The need to develop a new roughness measurement non-contact method arose when especially the artificially created roughness measurement of machined elements had to be carried out.

Thus, the technique of the rough surface observation using optical image defocusing with subsequent frequency analysis of a photoelectric signal has been proposed.

\section{PHYSICAL BASIS OF A NEW SURFACE FORMATION}

Formation of a new machined surface represents an engineering-technological process, which takes place under difficult physical conditions given by an immediate effect of a cutting tool - knife - on the surface layer of the workpiece to a certain depth while removing the surface layers. A process of chip formation in terms of creating the new surface, including the surface quality characterization [1], [8], [9], [10], [11], [12], has been studied by many authors. A principle of the new surface generation of the workpiece is schematically illustrated in Fig.1.

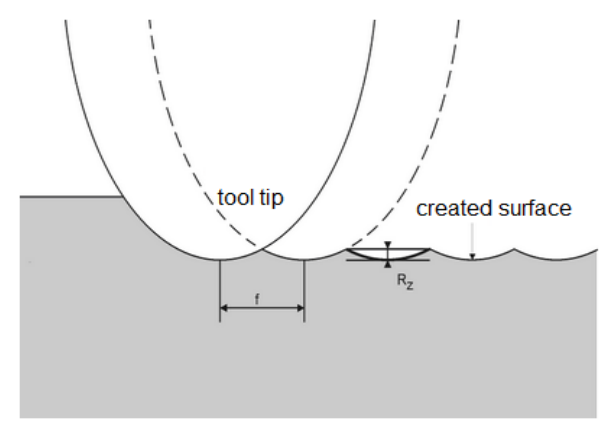

Fig.1. Schematic representation of interaction between the cutting tool tip and the formed surface. 
The theoretical surface roughness at lower feed rates can be defined by the tip radius of the cutting tool using well known geometrical relation [5]

$$
R_{Z}=\frac{f^{2}}{8 r_{\varepsilon}},
$$

where $r_{\varepsilon}$ is the tip radius of the tool [mm] and $f$ is the feed [mm] (see Fig. 1).

Although the illustration in Fig. 1 is a simplification of the process of the new workpiece surface formation, it represents its basic parameters. The mechanism of chip and new surface formation could therefore be considered only from the physical point of view, as a process that takes place within a force and stress-strain relationship and is concentrated in time and space in a zone near the cutting edge with its parameters. These parameters are characterized by the immediate action - reaction interaction of forces, tool tip shape, tool tip material, workpiece material and machining technology.

Some authors [1], [2], [8] ascribe the differences in the theoretical and actual surface roughness to a type of chip, which is formed during cutting. A discontinuous chip forms and separates from the workpiece in such a way that produces considerable surface undulations and cracks. This phenomenon leads to the cutting forces fluctuation and tool vibrations. Similar deterioration may arise with the continuous chip formation under such cutting conditions, which can lead to the formation of a built up edge on the tool tip. The built up edge arises as a result of physicalchemical compaction of the material in the contact area of the tool tip and affects the chip formation process, quality of the new surface, and tool cutting edge state.

The surface layers of the material are submitted to changes near the cutting edge. Deformation of grains is caused by the effect of the cutting tool and this deformation is completed by their fracture [8]. The type and character of the fracture depends on the material being machined, its crystalline structure and conditions of deformation, which are mainly determined by cutting conditions. In most cases, the fracture growth is carried out by a slip mechanism (dislocation process) or twinning. The resulting fracture surface must be assessed in terms of loading forces and stresses affecting the process of fracture formation, as well as in terms of the energy required, including the initial fracture velocity and fracture surface morphology.

\section{OPTICAL OBSERVATION OF SURFACE PROFILE}

Optical principle of monitoring the surface profile is based on the idea that a surface is composed of specularly reflecting surface facets and such a surface can be regarded as a phase object composed of phase inhomogeneities. When the surface is illuminated by a beam of collimated light, there is a light reflected specularly off the metallic surface. Regarding the mirror-like reflection, the phase difference between points on the rough surface can be observed, e.g., by a method of shadow visualization. Simple realization of the optical scheme is a projection of surface image with small defocusing to actual plane of observation.
The principal realization of the measurement follows from a specific character of the artificially formed surface roughness. Such character of the surface micro-profile is as a rule common in engineering technology because it is based upon basic machining technology principles. Machining grooves or scratches are formed by machine cutting tools and are more or less regularly spaced in one direction. The machined profile is thus periodically repeated with a dominant cylindrical shape due to finite radius of the cutting tool tip. The surface standards used in the experiment were planed and ground (sample VP 1 - 4).

Reading the light distribution after being reflected from the surface of the object and subsequent projection at the plane of observation was experimentally carried out by scanning of the projected image. The photodiode was used as a light detector. The effective photodiode area was shaded by a slit with the width of $0.2 \mathrm{~mm}$. The plane at the level of slit was situated at short distance $l$ from the imaging plane, as it is shown in Fig.2. For small defocusing, at the plane $I$ there is the "phase visualization" of specularlyreflective surface profile, having their roughness - waviness much larger than the wavelength of the light used [10]. This phenomenon follows from the fact that in the case of defocusing, the inverse Fourier transform is not fully accomplished. In order to get an expression for light distribution we have to derive the complex amplitude of the light $t_{l}(x)$ at the plane $I$, which is located behind the lens $L$ at a distance $l \neq q$.

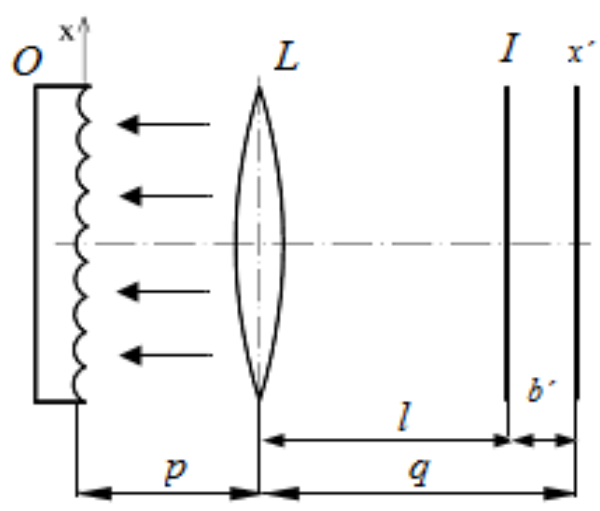

Fig.2. Scheme of phase visualization by defocusing.

The symbols used in Fig. 2 are as follows: $O-$ is the sample illuminated by a beam of collimated light, $L-$ is the lens projecting an image of the sample surface at distance $q$, $I-$ is the screen at a distance of $l \neq q$ from the lens $L, l-$ is the defocusing distance, $p, q$ - are subject and image distances, respectively.

Provided that the parameter $b$ is introduced

$$
b=\frac{\lambda}{4 \pi}(q-l)
$$

$T(\omega)$ is the Fourier image of a phase object $t(x)$, then 


$$
t_{1}(x)=\frac{1}{2 \pi} \int T(\omega) \exp (i \omega x) \exp \left(i b \omega^{2}\right) d \omega .
$$

If $b$ is sufficiently small, we can take only the first members of the development

$$
\exp \left(i b \omega^{2}\right) \approx l+i b \omega^{2},\left(b \Omega^{2}<<1\right)
$$

where $\Omega$ is the maximum spatial frequency at the image plane. Then,

$$
\begin{aligned}
& t_{1}(x)=\frac{1}{2 \pi} \int T(\omega) \exp (i \omega x)\left[1+i b \omega^{2}\right] d \omega= \\
& =t(x)+\frac{i b}{2 \pi} \int T(\omega) \omega^{2} \exp (i \omega x) d \omega
\end{aligned}
$$

Taking into account one of the properties of the Fourier transform

$$
\int T(\omega) \omega^{2} \exp (i \omega x) d \omega=-\frac{d^{2} t(x)}{d x^{2}},
$$

the complex amplitude of the defocused image is as follows

$$
t_{1}(x)=t(x)-i b \frac{d^{2} t(x)}{d x^{2}} .
$$

For the mirror-like surface as a phase object it can be written

$$
t(x)=\exp [i \phi(x)]
$$

and

$$
\frac{d^{2} t(x)}{d x^{2}}=\exp [i \phi(x)]\left\{i \frac{d^{2} \phi(x)}{d x^{2}}-\left(\frac{d \phi(x)}{d x}\right)^{2}\right\},
$$

or

$$
t_{1}(x)=t(x)\left\{1+b \frac{d^{2} \phi(x)}{d x^{2}}-i b\left(\frac{d \phi(x)}{d x}\right)^{2}\right\}
$$

Finally, the intensity distribution in the plane of the defocused image of the object is

$$
I(x)=\left|t_{1}(x)\right|^{2} \approx 1+2 b \frac{d^{2} \phi(x)}{d x^{2}} .
$$

Eq.(11) shows that at relatively small defocusing, the intensity distribution can be interpreted as a visualization of the second derivative of the surface profile. As known, the function of the second derivative is directly proportional to the radius of curvature of the specularly reflecting surface. A magnitude and sign of the image intensity distribution are given by the coefficient $b$. The value $b$ depends on the defocusing distance $l$ and the wavelength of light.

\section{EXPERIMENTAL MEASUREMENT AND RESULTS}

The measuring system based on scanning of the surface image for detection of surface artificial roughness of the surface profile is schematically shown in Fig.3. A collimated laser beam $(\phi=3.0 \mathrm{~mm})$ impinges the measured sample surface, which is moving approximately perpendicularly to the direction of grooves on the observed surface by a defined speed of $1.05 \mathrm{~m} \cdot \mathrm{s}^{-1}$. The incident beam is reflected from the uneven surface towards the objective according to the law of reflection and passes through a slit located in the vicinity of the image plane. The light intensity distribution is detected by a photodetector. Scanning is carried out by the movement of the sample. The optical signal observed is then converted into an electrical one and amplified. Thus, the time dependent oscillating variable of the intensity presents the value that is proportional to the intensity distribution. The electrical signal is recorded by a digital oscilloscope and transmitted into a PC. Post processing of spectral decomposition is obtained by Fast Fourier Transform (FFT) software. The frequency spectra can be converted into a spatial frequency spectrum for known speed of the sample, or to lateral dimensions (periodicity) of the surface roughness.

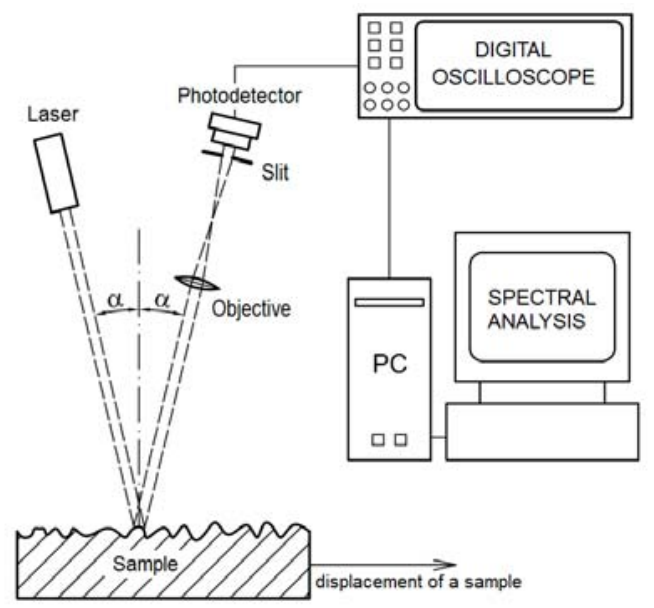

Fig.3. Scheme of the measurement system.

In the experiment Nd: YAG laser with a wavelength of $532 \mathrm{~nm}$ and output power of about $50 \mathrm{~mW}$ has been used as an illumination source. The sample surface was illuminated by non-expanded beam with a diameter of about $3.0 \mathrm{~mm}$ and the image of the illuminated area of the surface was projected through the lens with an aperture of 1:1.4 and a focal length $f=85 \mathrm{~mm}$. The projected image was enlarged approximately up to 2.5 times in the plane of the slit of the photodetector. Measurements were carried out on 12 standard samples V1 - V12, which were included in the Czech standard set of samples VP1 - 4. A key value of pitch separating waviness $(s)$ was determined in accordance with the measured frequency of sample surface. To compare the results, the samples were also measured in the laboratory by using a polarizing microscope Olympus BX60. The images recorded by three-chip Hitachi camera HV- C20 were evaluated by the image analysis software LUCIA G developed by Laboratory Imaging. 
The example of one of the surface images is shown in Fig.4. In Fig.5 there is a typical oscillating time dependence of the signal recorded by the photodetector. The corresponding frequency spectrum is demonstrated in Fig.6. Fig. 7 shows the correlation graph. To confront the values determined by experimental evaluation with those of standards, in Table 1 there are listed columns of mutual matching. As it can be seen from the values of the table, as well as from the graphical representation of Fig.7, there is good coincidence between the standard values $R a$ (column 3 ) and the values of $R a_{v}$ evaluated from results of the measurements (column 6). Moreover, close correlation is also shown between the values of parameter S (column 9) obtained by observations using polarizing microscope and the parameter $R a_{t}$ (column 7). Parameter $R a_{t}$ was calculated according to the surface roughness predicting equation (1) commonly used in mechanical machining [5] and the parameter $\mathrm{R}$ according to the microscopic data (column 10).

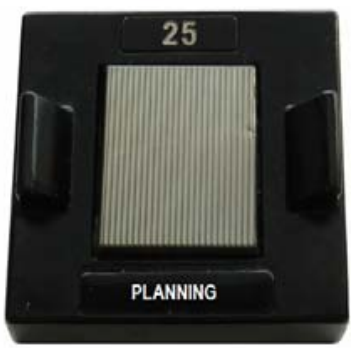

Fig.4. Machined surface produced by planning V9.

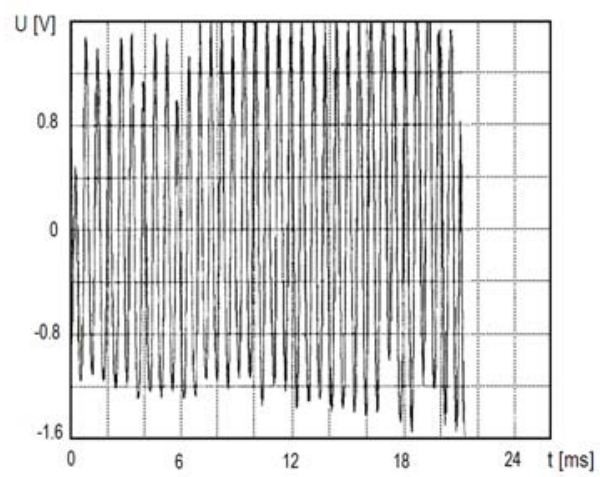

Fig.5. Time record of the optically scanned signal of the intensity of light reflected off the sample surface V9.

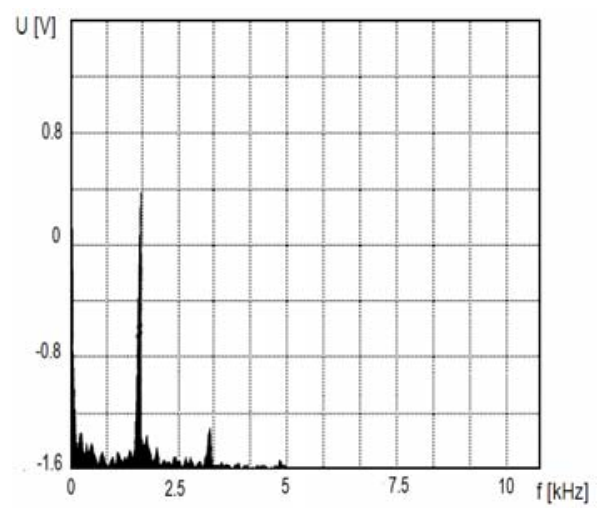

Fig.6. Frequency spectrum of the optical signal reflected off the sample surface V9.
Tab.1. Measurement results and their comparison with the values of standards.

\begin{tabular}{|c|c|c|c|c|c|c|c|c|c|}
\hline 1 & 2 & 3 & 4 & 5 & 6 & 7 & 8 & 9 & 10 \\
\hline no. SA. & Technology & $\begin{array}{c}R a \\
{[\mu \mathrm{m}]}\end{array}$ & $\begin{array}{c}f_{F} \\
{[\mathrm{kHz}]}\end{array}$ & $\begin{array}{c}s \\
{[\mathrm{~mm}]}\end{array}$ & $\begin{array}{c}R a_{v} \\
{[\mu \mathrm{m}]}\end{array}$ & $\begin{array}{c}R a_{t} \\
{[\mu \mathrm{m}]}\end{array}$ & $\begin{array}{c}f_{\text {KONIR }} \\
{[\mathrm{kHz}]}\end{array}$ & $\begin{array}{c}S \\
{[\mathrm{~mm}]}\end{array}$ & $\begin{array}{c}R \\
{[\mu \mathrm{m}]}\end{array}$ \\
\hline V1 & FCGR & 0.1 & 6.67 & 0.16 & 0.50 & 0.31 & 33.6 & 0.024 & 0.08 \\
\hline V2 & FCGR & 0.2 & 16.67 & 0.06 & 0.20 & 0.19 & 16.8 & 0.159 & 0.51 \\
\hline V3 & FCGR & 0.4 & 7.92 & 0.14 & 0.45 & 0.41 & 8.4 & 0.375 & 1.20 \\
\hline V4 & FCGR & 0.8 & 4.58 & 0.23 & 0.73 & 0.78 & 4.2 & 0.492 & 1.57 \\
\hline V5 & FCGR & 1.6 & 1.83 & 0.57 & 1.82 & 1.72 & 2.1 & 0.331 & 1.06 \\
\hline V6 & PL SC & 1.6 & 6.5 & 0.16 & 3.20 & 2.71 & 13.1 & 0.123 & 2.46 \\
\hline V7 & PL HS & 6.3 & 3.33 & 0.32 & 6.40 & 6.70 & 3.33 & 0.372 & 7.44 \\
\hline V8 & PL SC & 12.5 & 1.63 & 0.64 & 12.88 & 13.12 & 1.68 & 0.632 & 12.46 \\
\hline V9 & PL SC & 25 & 0.79 & 1.33 & 26.53 & 26.45 & 0.83 & 0.863 & 17.26 \\
\hline V10 & FCGR & 1.6 & 2.00 & 0.53 & 1.68 & 1.78 & 2.1 & 0.628 & 2.01 \\
\hline V11 & PL HS & 6.3 & 3.25 & 0.32 & 6.46 & 6.70 & 3.33 & 0.372 & 7.64 \\
\hline V12 & PL SC & 12.5 & 1.67 & 0.63 & 12.60 & 13.72 & 1.68 & 0.632 & 12.46 \\
\hline
\end{tabular}

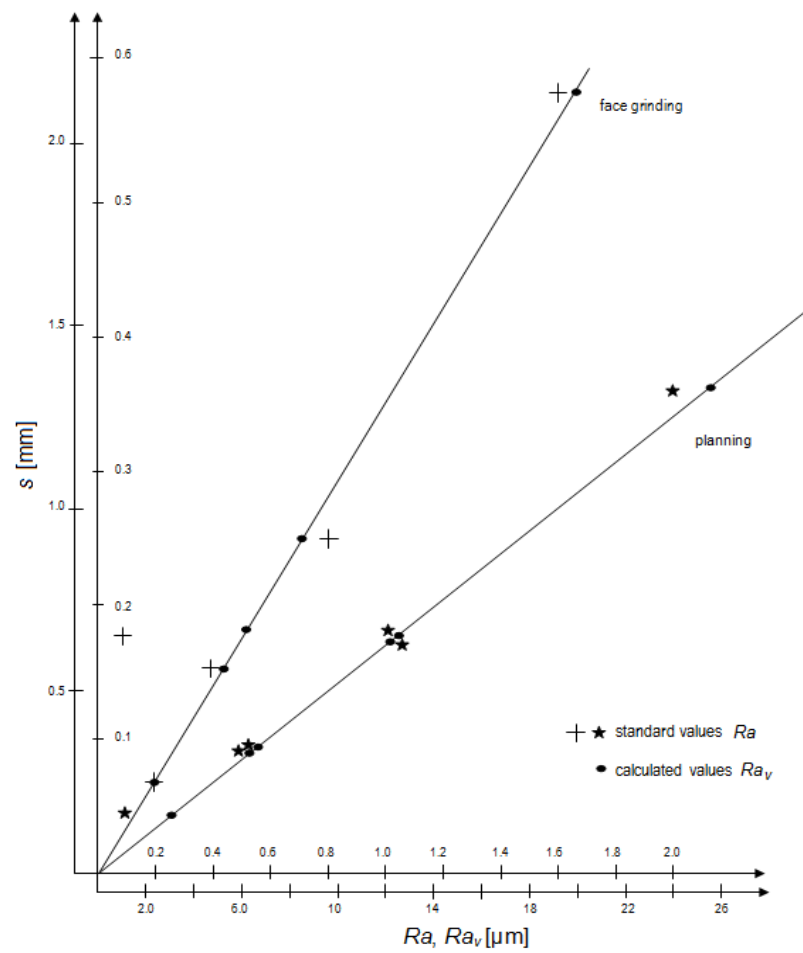

Fig.7. Dependence of the standard surface roughness $R a$ and the calculated surface roughness $R_{a t}$ at a certain pitch $s$ of adjacent traces left behind the machine tool.

When plotted as a control chart, the functional dependences of $R a(s)$ were obtained for two different mechanical surface treatment technologies used at the process of fabrication of etalon samples. The samples V1 to V5 and the sample V10 were machined by face crosswise grinding (FCGR) and the rest was machined by planning (PL). The method has been approved reliable and sensitive enough to acquire the qualitative differences of surface finishing methods being used on standard samples. For the quantitative determination of the measured parameters, there were found the linear correlation functions of the form 
or

$$
R a_{v}=k_{F C G R} \cdot s,
$$

$$
R a_{v}=k_{P L} \cdot s,
$$

with the coefficients of proportionality for $k_{F C G R}$ of 3.20 and for $k_{P L}$ of 20 . The parameter $s$ was evaluated from the measured function $f_{A}$, where $f_{A}$ is the frequency obtained from the frequency-amplitude spectrum.

\section{CONCLUSION}

The frequent utilization of speckle techniques for measuring surface roughness is, as a rule, limited to stochastic surfaces whose roughness is relatively low approximately in the range of wavelengths of light. Moreover, the artificially created surfaces ask for some specific measurement requirements after machining. That is why the optical diagnostics method and relevant measuring system have been proposed and experimentally carried out with the aim to fulfill the demands for complete characterization, particularly of the metallic surfaces. By using the newly developed method, the results were acquired that indicated a good coincidence of the surface roughness values obtained by such an approach with those assessed from microscopic measurements. Analysis of both actual objective and subjective errors associated with the method of measurement and the way of its interpretation has been performed. The method proposed has shown the feasibility to determine the local radii of curvature of the surface profile formed on the surface by the machining tool sharp tip. With regard to simple scheme realization as well as general advantages of the optical measuring principle such as non-contact measurement, the setup robustness and simple adjustment by user with no special requirements to the device fixation, the designed profilometry tool seems to be useful and perspective for practical implementation. By comparing the roughness standard values $R a$, the relationship was obtained between both the values of $R a$ and the parameter of characteristic frequency of vertical unevenness measured according to the presented method. As it was approved by experiments, high degree of coincidence has been obtained between the optically determined parameters and those of surface standard values. Correlation coefficient is shown to be in the range of 0.8 to 1 .

\section{ACKNOWLEDGMENT}

This work was supported by the projects MoravianSilesian region No. 0014/2012/RRC, RMTVC No. CZ.1.05/2.1.00/01.0040, ICT No. CZ.1.05/2.1.00/03.0082, IT4Innovations Centre of Excellence project, reg. no. CZ.1.05/1.1.00/02.0070 and Research and Development Agency (contract APVV-0032-10), Slovak Republic.

\section{REFERENCES}

[1] Whitehouse, D.J. (1997). Surface metrology. Measurement Science and Technology, 8 (9), 955-972.

[2] Thomas, T.R. (1982). Rough Surfaces. London: Longman.

[3] Ohlidal, M. (1995). Comparison of 2-dimensional Fraunhofer approximation and 2-dimensional Fresnel approximation at analysis of surface-roughness by angle speckle correlation. 2. Experimental results. Journal of Modern Optics, 42 (10), 2081-2094.

[4] Nadolny, K. et al. (2011). Laser measurements of surface topography of abrasive tools using measurement system CLI 2000. Przeglad Elektrotechniczny (Electrical Review), 87 (9a), 24-27.

[5] Lukianowicz, C., Karpiński, T. (2001). Optical system for measurement of surface form and roughness. Measurement Science Review, 1 (1), 151-154.

[6] Senchenko, E.S., Chugui, Yu.V. (2011). Shadow inspection of 3D objects in partially coherent light. Measurement Science Review, 11 (4), 104-107.

[7] Kawate, E., Hain, M. (2012). New scatterometer for spatial distribution measurements of light scattering from materials. Measurement Science Review, 12 (2), 56-61.

[8] Bumbalek, B. (1983). Statistical-analysis and its use evaluating surface microgeometry. Kovove Materialy Metallic Materials, 21 (6), 687-698.

[9] Geleta, V., Mészároš, I. (2001). Surface roughness formation of some mechatronic parts. In 4th International Symposium on Mechatronics Mechatronika, 13-15 June 2001.

[10] Ogilvy, J.A. (1991). Theory of Scattering from Random Rough Surfaces. IOP Publishing.

[11] Lukianowicz, C. (2003). Modeling of direct and inverse problems in light scattering by rough surfaces. Optica Applicata, 33 (2-3), 329-336.

[12] Kapłonek, W., Łukianowicz, C. (2008). Assessment of surface microroughness in movement by laser scatterometry and image stacking. Przeglad Elektrotechniczny (Electrical Review), 84 (5), 155-160.

Received July 10, 2012. Accepted September 14, 2012. 